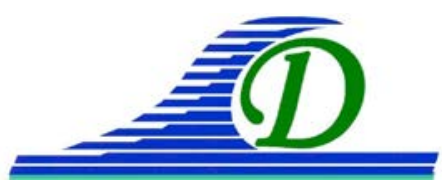
XIII ${ }^{\text {èmes }}$ Journées Nationales Génie Côtier - Génie Civil Dunkerque, 2-4 juillet 2014

DOI:10.5150/jngcgc.2014.067 @ Editions Paralia CFL

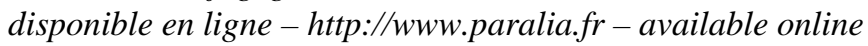

\title{
RIVERCOLOR : chaîne de traitement des séries temporelles LANDSAT, SPOT et MODIS dédiée à la cartographie des matières en suspension en zone estuarienne
}

\author{
Virginie LAFON ${ }^{1}$, Arthur ROBINET ${ }^{1}$, Tatianna DONNAY ${ }^{2}$, David DOXARAN ${ }^{2}$, \\ Bertrand LUBAC ${ }^{3}$, Eric MANEUX ${ }^{1}$, Aldo SOTTOLICHIO ${ }^{3}$, Olivier HAGOLLE ${ }^{4}$ \\ 1. GEO-Transfert, UMR 5805 EPOC, ADERA, allée Geoffroy St Hilaire, \\ CS 50023, 33615 Pessac cedex, France. \\ v.lafon@epoc.u-bordeaux1.fr \\ 2. Laboratoire d'Océanographie de Villefranche, UMR 7093 - CNRS / UPMC, \\ 181 chemin du Lazaret, 06230 Villefranche-sur-Mer, France. \\ 3. UMR 5805 EPOC, allée Geoffroy St Hilaire, CS 50023, 33615 Pessac cedex, France. \\ 4. CESBIO, 18 avenue Edouard Belin, bpi 2801, 31401 Toulouse cedex, France.
}

\section{Résumé :}

Cette contribution conclut la première année du projet RIVERCOLOR dont l'objectif est de livrer des cartes de matières en suspension (MES) de surface des eaux estuariennes, dérivées d'images satellite à haute et moyenne résolution spatiale. Ces travaux décrivent la méthode suivie pour définir des algorithmes de détection des MES pour les capteurs LANDSAT, SPOT et MODIS. Des bases de données de match-ups sont constituées pour éprouver leur robustesse. La corrélation entre les jeux de données est très significative $\left(\mathrm{R}^{2}>0,8\right)$ et les variations des MES satellites reflètent correctement les variations observées par les stations, malgré un biais marqué entre les MES satellites et les MES in situ. Les principales étapes de la chaîne de traitement d'images développée sont également détaillées, en particulier la sélection des données, le traitement des nuages et de leurs ombres sur l'eau. Enfin, un bilan chiffré des images exploitables pour les dernières années d'acquisition est présenté.

Mots-clés: Turbidité, Gironde, Estuaire, Matière en suspension, Inversion, Optique, Télédétection.

\section{Introduction}

Les grands estuaires présentent une zone de turbidité naturelle très marquée (le bouchon vaseux) qui a un impact prépondérant sur les bilans sédimentaires, les processus biogéochimiques et la qualité biologique des milieux (oxygénation). Les observations satellitaires de la couleur de l'eau constituent une source de données indispensable à la connaissance spatio-temporelle de la variabilité naturelle de la turbidité des eaux. Dans le cas de la Gironde, la faisabilité de la mesure des matières en suspension (MES) à partir de données SPOT et MODIS a été démontrée (DOXARAN et al., $2002 \&$ 2003). Ces observations satellitaires complètent le suivi MAGEST (ETCHEBER et al., 2010 ; 
SOTTOLICHIO et al., 2011) en spatialisant les mesures en MES. Elles apportent en outre une vision dynamique du déplacement des masses turbides, en particulier en lien avec les variations du bouchon vaseux (DOXARAN et al., 2009, figure 1).

Dans ce contexte, le projet RIVERCOLOR vise notamment deux objectifs autour desquels s'articule la présente contribution : (i) validation d'algorithmes permettant de décrire la distribution spatiale des MES dans l'estuaire de la Gironde à partir de données satellitaires (produits hautes et moyenne résolution spatiale comme SPOT, LANDSAT, MODIS et MERIS); (ii) développement d'une chaîne de traitement du produit MES adaptée aux principaux capteurs optiques à moyenne et haute résolution spatiale actuels et futurs (Sentinel-3 et -2). La diffusion régulière de cartes de MES, basée sur l'acquisition d'images hebdomadaires (Sentinel-2) à quotidiennes (Sentinel-3), permettra d'améliorer la connaissance de la dynamique du bouchon vaseux et du panache et notamment de contribuer à l'évaluation des flux particulaires sortants de l'estuaire, mais également de caractériser l'hétérogénéité spatiale de la qualité des eaux de l'estuaire et de participer à la validation des modèles hydro-sédimentaires (SiAM 3D dans la Gironde).

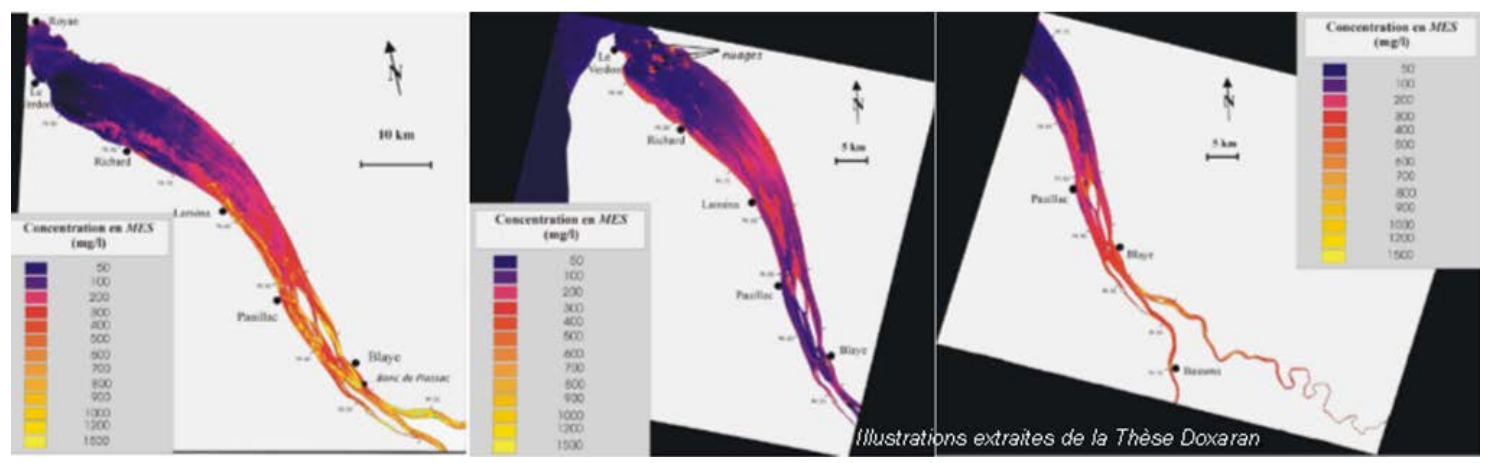

Figure 1. Carte des concentrations en MES de surface dans la Gironde par imagerie MODIS (d'après DOXARAN, 2002). De gauche à droite : débit moyen, crue, étiage.

\section{Données et méthodes}

\subsection{Données de terrain}

Le réseau MAGEST est basé sur le déploiement de 4 stations automatisées Marel (WOERTHER \& GROUHEL, 1998) qui mesurent en continu, toutes les 10 minutes, température, salinité, turbidité et taux d'oxygène dissous des eaux estuariennes. Les stations sont situées à Pauillac, Bordeaux, Portets et Libourne, sur les berges du fleuve, en bordure d'un quai.

Des campagnes de mesure des propriétés apparentes à la surface de l'eau ont été entreprises depuis 2012 afin de mieux comprendre le comportement de la réflectance de l'eau en fonction de la turbidité et de simuler les capteurs satellites. Ces campagnes 


\section{XIII ${ }^{\text {èmes }}$ Journées Nationales Génie Côtier - Génie Civil \\ Dunkerque, 2-4 juillet 2014}

mettent en œuvre trois capteurs optiques TriOS ${ }^{\circledR}$ selon le protocole édité par la NASA (Ocean Optics Protocols For Satellite Ocean Color Sensor Validation, Revision 4, Volume III : Radiometric Measurements and data Analysis Protocols (2003)). Chaque mesure de réflectance est accompagnée d'un prélèvement d'eau qui est filtré afin de mesurer la concentration en matières en suspension totale (MES). Ce jeu de données permet d'établir des corrélations entre réflectance de l'eau et MES.

Sur les trois capteurs TriOS, deux mesurent des luminances et le troisième mesure un éclairement. Le capteur d'éclairement est orienté vers le zénith tandis que les capteurs de luminance sont orientés selon un angle de $45^{\circ}$, l'un vers l'eau, le second en direction $\mathrm{du}$ ciel. Les instruments sont positionnés à $135^{\circ} \mathrm{du}$ plan du Soleil. Le capteur d'éclairement $(E d)$ mesure le rayonnement lumineux sur l'ensemble d'une demi-sphère. Le capteur de luminance ( $7^{\circ}$ d'ouverture) dirigé vers l'eau mesure $L_{w}$ et celui dirigé vers le ciel mesure la luminance émise par l'atmosphère (notée $\mathrm{L}_{\mathrm{sky}}$ ).

Chaque capteur mesure l'intensité lumineuse aux longueurs d'onde comprises entre 350 et $900 \mathrm{~nm}$ avec une résolution de $2,5 \mathrm{~nm}$. Chaque échantillonnage correspond à cinq mesures espacées de 10 secondes qui sont moyennées. A partir de ces mesures, nous calculons une réflectance de surface grâce à la relation $\mathrm{R}_{\mathrm{rs}}=\left(\mathrm{L}_{\mathrm{w}}-\rho \times \mathrm{L}_{\mathrm{sky}}\right) / \mathrm{E}_{\mathrm{d}}$, où $\rho$ est la réflectance de Fresnel à l'interface air-mer. Il correspond au pourcentage de la luminance du ciel directement réfléchi par la surface. Dans notre étude $\rho$ est égal à soit 0,0256 soit la valeur moyenne en considérant la surface de l'eau plate (pas de vague) et un angle zénithal de $30^{\circ}$ (MUELLER et al., 1997). Au total, 60 spectres ont été acquis suivant cette approche. Les bandes SPOT et LANDSAT ont été simulées grâce à ces mesures en tenant compte de la sensibilité spectrale des capteurs.

\subsection{Données satellites}

Les images MODIS ont été téléchargées pour une période comprise entre mai 2008 et juillet 2009 depuis les sites reverb.echo.nasa.gov et oceancolor.gsfc.nasa.gov pour les produits SR et Rrs, respectivement. Le produit Rrs, de niveau 1, est corrigé des effets atmosphériques à l'aide du logiciel SeaDAS en appliquant la correction atmosphérique PIR-SWIR (WANG et al., 2009). SR, de niveau 2, est livré en réflectance de surface. Les bande proche infrarouge (PIR, $859 \mathrm{~nm}$ ) et rouge $(\mathrm{R}, 645 \mathrm{~nm})$ d'une résolution spatiale de $250 \mathrm{~m}$ ont été retenues pour cette étude. Du fait de cette faible résolution spatiale, nous nous concentrerons pour les données MODIS sur les données MAGEST de Pauillac. Parmi les produits téléchargés (365), les 28 images satellites ne présentant aucune couverture nuageuse dans le secteur de Pauillac et acquises lors de périodes aux cours desquelles la station MAGEST était en fonctionnement ont été sélectionnées.

Pour la haute résolution, nous avons testé deux bases de données images: SPOT4 (Take5) Aquitaine (6 vues entre janvier - juin 2013) et LANDSAT 5 et 7 acquises en 2011 (BD TOSCA LANDSAT : 13 vues au total) traitées par le CESBIO à partir d'une chaîne de traitement commune (HAGOLLE et al., 2010). Les produits sont ortho- 


\section{Thème 3 - Instrumentation, mesures, imagerie et télédétection}

rectifiés et projetés (Lambert 93), livrés en réflectance de surface corrigée des effets atmosphériques (incluant les effets d'environnement et de pente). La résolution des images SPOT4 est de $20 \mathrm{~m}$ au sol, celle de LANDSAT de $30 \mathrm{~m}$. Au total, 13 scènes LANDSAT et 5 scènes SPOT4 sont disponibles et exploitables réparties de façon homogène dans le temps. Sur ce nombre total de scènes (tous capteurs confondus), seuls quelques match-ups peuvent être obtenus pour les différentes stations du réseau MAGEST : 6 à Pauillac, 12 à Bordeaux, 9 à Portets et 5 à Libourne. Pour l'analyse des données SPOT et LANDSAT, nous nous concentrerons sur les données de Bordeaux, plus nombreuses et aussi plus représentatives de saisons hydrologiques contrastées.

\subsection{Analyse de sensibilité des capteurs aux variations de MES}

Pour définir des algorithmes d'inversion de la mesure de réflectance en MES, des analyses sont menées, basées sur la comparaison entre les mesures de turbidité in situ et les données satellites. Des bases de données d'observations (terrain et satellites) colocalisées (match-ups) sont constituées comme suit. Pour chaque image disponible sur une période d'une année (2008-2009 pour MODIS, 2011 pour LANDSAT, 2013 pour SPOT), les valeurs des pixels dans une fenêtre de $3 \times 3$ sont extraites au large des stations MAGEST de Pauillac (MODIS) et Bordeaux (LANDSAT et SPOT). Celles-ci sont situées sur les berges mais seuls les pixels non contaminés par la présence de ces dernières (préalablement masquées) sont utilisés pour obtenir une corrélation avec les mesures satellites. Neuf corrélations sont établies entre la mesure MAGEST et les neufs pixels de la fenêtre, en considérant chacun des produits image et chacune des bandes spectrales de ces produits. Les résultats sont comparés afin de déterminer le meilleur produit à partir des régressions établies pour chaque relation obtenue. Cette analyse permettra en outre d'analyser la relation spatiale entre mesure MAGEST et donnée image, car nous ne pouvons établir de corrélation entre deux variables parfaitement colocalisées. Cette stratégie a été appliquée aux deux produits MODIS (niveau 1 corrigé des effets atmosphériques et niveau 2 livré en réflectance de surface), ainsi qu'aux images LANDSAT et SPOT4.

\subsection{Algorithmes de MES : conception et validation}

Un algorithme publié par DOXARAN et al. (2009) est adapté aux données MODIS et relie par une loi exponentielle les MES au rapport des réflectance PIR (proche infrarouge) sur $\mathrm{R}$ (rouge). Cet algorithme a été obtenu avec des mesures de terrain de réflectance et MES simultanées. La même approche a été utilisée pour obtenir un algorithme de détection des MES à partir des données à très haute résolution spatiale en exploitant les campagnes de mesure hyperspectrale décrites dans le paragraphe 2.1. Les données hyperspectrales (Trios) acquises sur le terrain ont permis de simuler les canaux des capteurs LANDSAT et SPOT. Le canal le plus sensible aux variations de MES déduit de l'analyse de sensibilité des capteurs aux variations de MES a été sélectionné 


\section{XIII ${ }^{\text {èmes }}$ Journées Nationales Génie Côtier - Génie Civil \\ Dunkerque, 2-4 juillet 2014}

parmi les 4 canaux ou compositions de canaux possibles (R, PIR, R/PIR, PIR/R). Puis une corrélation empirique a été établie entre l'algorithme retenu et les MES simultanément mesurées sur le terrain. Cet algorithme empirique est enfin appliqué aux images et les MES satellites sont comparées aux MES MAGEST pour valider l'algorithme d'inversion établi pour les images à haute résolution spatiale.

\subsection{Chaîne de traitement}

La chaine de traitement est développée en premier lieu pour le produit MODIS le plus adapté à la mesure de MES (SR ou Rrs, à définir). Elle est conçue pour être adaptée à d'autres types de produit/capteur à condition qu'un algorithme d'inversion correspondant soit disponible. La chaine de traitement consiste en une succession d'étapes permettant la génération de carte de MES sur l'estuaire, après (1) vérification de l'exploitabilité des produits, (2) application d'un masque de terre et (3) détection des nuages et de leurs ombres.

En fonction des données images à intégrer, d'autres étapes de traitement sont à prévoir. Par exemple, les produits L2 de MODIS (SR) sont fournis pour une emprise spatiale constante mais dont la dimension caractéristique dépasse celle de la largeur de la fauchée du capteur. Ainsi, 2 fauchées séparées d'au plus $1 \mathrm{~h} 30$ peuvent être utilisées pour constituer le produit L2 de MODIS couvrant l'estuaire de la Gironde. La valeur de chaque pixel affecté par un chevauchement de plusieurs fauchées est celle appartenant à la fauchée ayant permis la meilleure observation en terme de qualité. Le chevauchement de fauchée produit ainsi des zones composites sur les images, où l'analyse de processus rapides ne peut être effectuée. Il convient alors de rejeter les images pour lesquelles l'estuaire de la Gironde est affecté par un chevauchement de fauchée. Le nombre d'observations réalisées pour chacun des pixels de l'image étant fourni dans les produits L2 de MODIS (VERMOTE et al., 2011).

Le masque de terre est digitalisé à partir d'images obtenues à des dates où la hauteur d'eau dans l'estuaire est au plus bas afin d'éliminer toutes les berges qui pourraient contribuer à la réflectance de surface.

Pour la détection des nuages, dans le cas de séries temporelles d'images, l'utilisation d'une approche multi-temporelle est très efficace (HAGOLLE et al., 2010). Celle-ci s'appuie sur la forte stabilité des réflectances de surface comparée aux fortes variations causées par la présence de nuages pour des images obtenues à des dates proches. Cette méthode offre de meilleurs résultats que l'utilisation de seuils. Malheureusement, l'hypothèse de forte stabilité des réflectances de surface n'est pas vérifiée au-dessus des cours d'eau du fait des fortes variations temporelles de turbidité. Ces variations pourraient être interprétées comme la présence de couvert nuageux, conduisant alors à des échecs répétés de détection. Une approche alternative a été définie dans le cadre de notre étude en se basant sur des valeurs seuils (ex. DOXARAN et al., 2009). 
La chaîne RIVERCOLOR a enfin été exploitée sur les archives 2012 et 2013 pour extraire une statistique des images exploitables pour la cartographie temporelle des MES de la Gironde.

\section{Résultats et discussion}

\subsection{Sélection du produit MODIS}

Les comparaisons des deux produits MODIS à chaque longueur d'onde (645 et $859 \mathrm{~nm}$ ) et pour le rapport spectral 859/645 montrent plusieurs différences fondamentales entre les jeux de données.

Tout d'abord, les bandes rouges $(645 \mathrm{~nm})$ des produits Rrs et SR montrent au centre de l'estuaire des différences significatives sur le plans radiométrique, mais également spatial. En effet, le nombre de pixels disponibles pour une inversion est beaucoup moins important dans le cas des produits de niveau 1 (Rrs). Pour la bande PIR (859 nm) les pixels du produit Rrs sont masqués dans la partie amont de l'estuaire (valeurs négatives). En ce qui concerne le rapport spectral entre les deux longueurs d'onde, les produits Rrs et SR sont très similaires dans la partie aval de l'estuaire. Quant aux pixels qui entourent la station de Pauillac, les valeurs sont plus homogènes pour le produit SR que Rrs, qui montre une très forte variabilité spatiale. Après une étude sur les pixels autour de la station Marel, le pixel qui représente la meilleure corrélation entre la turbidité et la mesure satellite se trouve au sud-est de la station, à environ $750 \mathrm{~m}$. La corrélation entre MODIS SR (pixel distant de $750 \mathrm{~m}$ ) et MAGEST est présenté sur la figure 2. Le coefficient de corrélation est relativement faible. Ceci peut s'expliquer par l'éloignement spatial entre la mesure satellite et la mesure de terrain. Ainsi, si le produit satellite MODIS SR semble être le mieux adapté pour estimer la concentration en MES dans l'estuaire de la Gironde, une expérimentation en cours de réalisation vise à déterminer la représentativité spatiale de la station MAGEST, afin notamment de discuter et valider le protocole actuel de validation des MES spatiales par les mesures MAGEST.

\subsection{Produits MES à haute résolution spatiale}

La sensibilité des données haute résolution a été analysée tous capteurs confondus, car le nombre de match-ups est trop peu important pour faire une analyse capteur par capteur. Concernant le choix des bandes spectrales sensibles à la variabilité des MES, une meilleure corrélation est pour le rapport rouge/PIR $\left(\mathrm{R}^{2}=0,77, \mathrm{~N}=12\right)$ que pour le rapport vert/PIR $\left(\mathrm{R}^{2}=0,71\right)$. Ces bonnes corrélations prouvent tout d'abord les données SPOT et LANDSAT sont spectralement fortement inter-corrélées. Si la corrélation est $a$ priori meilleure pour le rapport rouge/PIR, en revanche, l'écart type sur les valeurs de réflectance dans le rouge autour du point de mesure est supérieur de $30 \%$ à celui calculé dans la bande verte. Cette analyse montre la très forte variabilité spatiale des 


\section{XIII ${ }^{\text {èmes }}$ Journées Nationales Génie Côtier - Génie Civil \\ Dunkerque, 2-4 juillet 2014}

réflectances à cette longueur d'onde peut-être alors moins propice à établir des MES avec une barre d'erreur acceptable. Pour vérifier ce point, des ajustements ont été réalisés entre MES et réflectances simultanées in situ pour les deux rapports de bande et pour simuler les trois capteurs LANDSAT 5 et 7 et SPOT 4 indépendamment. Six relations exponentielles sont ainsi obtenues toutes de bonne qualité $\left(R^{2}\right.$ compris entre 0,87 et $0,88, \mathrm{~N}=60$ ). Ces relations ont été appliquées aux 12 images pour lesquelles des données de validation de la station MAGEST de Bordeaux sont disponibles.

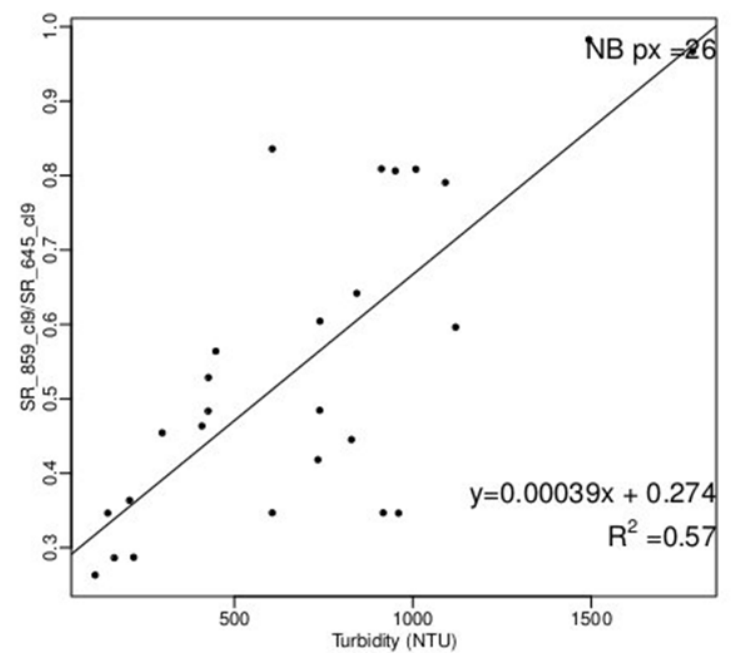

Figure 2. Match-ups entre le rapport SR_859 / SR_659 (mesures satellites) et la turbidité mesurée in situ (station Marel de Pauillac).

La comparaison entre les MES calculées et mesurées à Bordeaux montre que le rapport de réflectance rouge/PIR restitue un peu mieux les MES que le rapport vert/PIR. Dans les deux cas, les MES évaluées sont sous-estimées par rapport aux mesures Marel de Bordeaux (ex. figure 3). La sous-estimation atteint $390 \mathrm{mg} / \mathrm{l} \mathrm{en} \mathrm{moyenne} \mathrm{pour} \mathrm{le} \mathrm{rapport}$ rouge/PIR et $510 \mathrm{mg} / \mathrm{l}$ pour le rapport vert/PIR. Ce chiffrage reste peu significatif car la représentativité des stations Marel par rapport aux pixels SPOT et LANDSAT reste encore à établir. De plus, le nombre de match-ups est faible pour conclure ces travaux.

Toutefois, il est très intéressant de noter que les MES produites suivent les variations de celles mesurées (cf. figure 3 ), et ce avec un très bon degré de corrélation $\left(\mathrm{R}^{2}=0,89\right)$ au moins pour la gamme $0-2000 \mathrm{mg} / \mathrm{l}$. Au-delà, les deux plus fortes valeurs de MES mesurées par satellite correspondent à une saturation des capteurs de turbidité qui ont cessé de fonctionner. Les barres d'erreur sur les MES, calculées en comparant les mesures MAGEST et satellite à haute résolution de 2011 et 2013, sont très raisonnables (de l'ordre de $100 \mathrm{mg} / \mathrm{l}$ ) mais les écarts entre la mesure et satellite et la mesure de terrain peuvent atteindre ponctuellement plus de $600 \mathrm{mg} / \mathrm{l}$. Il est nécessaire de mieux comprendre la représentativité spatiale de la mesure MAGEST par rapport à la mesure satellite et vérifier que nous comparons des mesures qui simultanément sont réellement 
comparables. Une expérimentation réalisée dans ce but est en cours. La corrélation obtenue avec le rapport vert/PIR reste bonne $\left(\mathrm{R}^{2}=0,77\right)$.

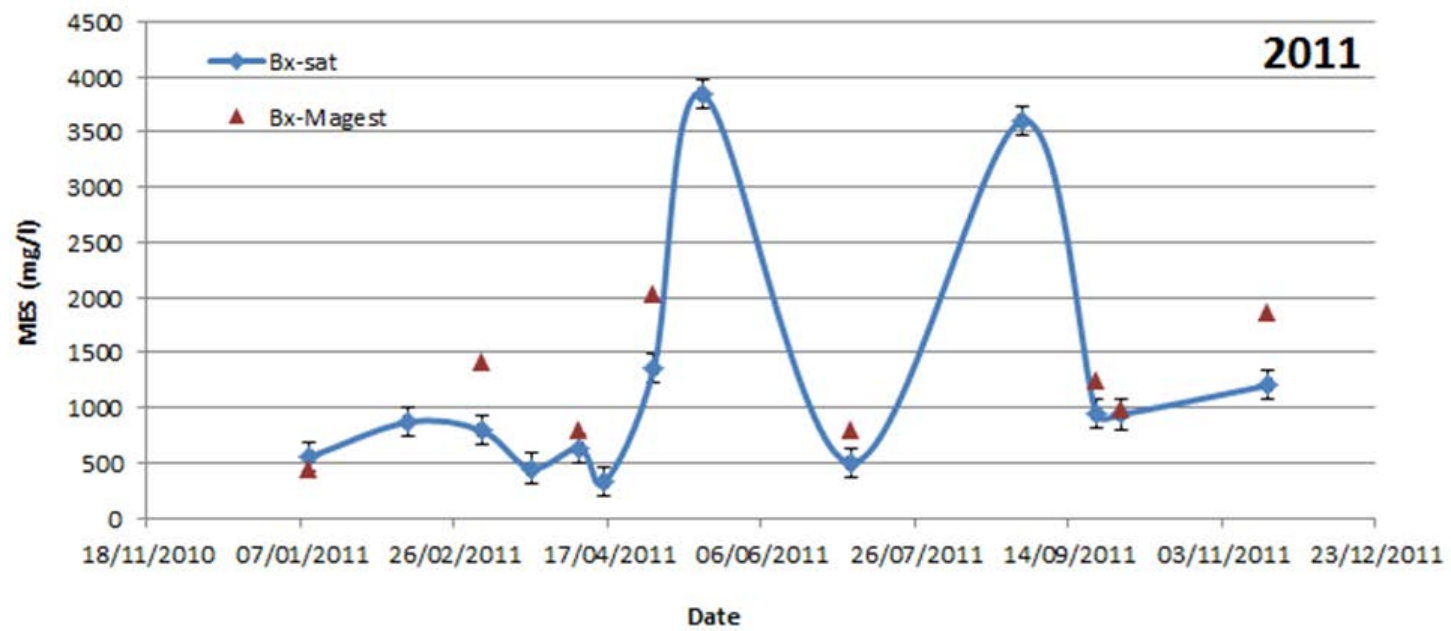

Figure 3. Comparaison MES mesurées / MES calculées pour les différentes acquisitions en 2011.

\subsection{Produits dérivés de la chaîne de traitement}

Dans un premier temps, la chaîne de traitement a été développée spécifiquement pour le produit MODIS AQUA de niveau 2. Outre le masque de Terre et l'élimination des images composites (en moyenne 1/3 en 2012 et 2013), les développements les plus poussés concernent la détection des nuages et de leurs ombres.

Au-dessus de zone océanique, qui ne rayonne en général que très peu dans la bande PIR, la détection de nuages par l'utilisation d'un seuil fournit de bons résultats. Cependant, la présence de MES dans l'eau augmente sensiblement les valeurs de réflectance mais ne conduit que très rarement à des réflectances de surface supérieures à 20\% dans la bande PIR pour l'image MODIS. Dans la chaîne de traitement développée, ce seuil de $20 \%$ est utilisé pour révéler les pixels nuageux. L'analyse du résultat de l'effet de l'application d'une dilatation des pixels nuageux permet de classifier la distribution des nuages et donc de renseigner sur le type de nuages présent. Si, après dilatation, la proportion de nuage augmente fortement alors les nuages sont de types épars, tandis que si la dilatation ne produit qu'une plus faible augmentation du nombre de pixels nuageux alors nous pouvons supposer que le couvert nuageux est plus compact. En fonction du nombre et de la distribution des pixels de nuage les images sont rejetées, ou traitées. Parmi les images traitées, celles dont la classification aurait pu être incertaine sont contrôlées visuellement.

La recherche de l'ombre des nuages est délicate car le produit SR MODIS ne contient pas les angles d'observations de la scène ni les angles solaires nécessaires au positionnement des ombres par rapport aux nuages détectés. En première approche, une 


\section{XIII ${ }^{\text {èmes }}$ Journées Nationales Génie Côtier - Génie Civil \\ Dunkerque, 2-4 juillet 2014}

alternative simple consiste à supposer que les réflectances de surface inférieures à une valeur seuil dans la bande rouge correspondent à celles des ombres de nuages. En effet, l'analyse d'histogrammes de réflectance de surface dans la bande rouge de nombreuses images impactées par des nuages et leurs ombres, montre que, généralement, les plus faibles réflectances correspondent aux pixels ombragés. La connaissance du nombre de pixels ombragés permet de lire dans l'histogramme des réflectances de surface la valeur seuil qui doit être prise pour masquer ces pixels, l'estimation du nombre de pixels ombragés dépendant essentiellement du nombre de pixels nuageux et de leur distribution sur l'estuaire de la Gironde. Cette méthode simpliste offre l'avantage de permettre la détection de la plupart des ombres sans nécessiter la connaissance de la géométrie de la prise de vue. Elle présente toutefois l'inconvénient de surestimer la quantité de pixels ombragés avec pour principale conséquence la classification des pixels de plus faible concentration en MES en pixel d'ombre. Ce problème se pose particulièrement lorsque les ombres des nuages sont en dehors de la zone non-masquée ou que l'estimation du nombre de pixels ombragés est trop élevée.

A l'issu de ce travail, au total sur les $2 / 3$ d'images restantes, $63 \%$ sont qualifiées trop nuageuses pour être exploitées. Les 27\% restantes (83 images en 2012 et 107 en 2013) montrent dans la grande majorité la présence de nuages plus ou moins nombreux. Une analyse visuelle des produits MES montre que le couvert nuageux a tendance à être sous-estimé ( $22 \%$ des cas) et les ombres plutôt surestimées ( $12 \%$ des cas).

Pour augmenter ce quota de données, les données composites doivent être analysées une par une afin de ré-intégrer les données exploitables.

\section{Synthèse des résultats}

En moyenne, dans une configuration proche des futures acquisitions Sentinel-2 (tous les 5 jours), une image par mois est exploitable sur l'estuaire de la Gironde, à haute résolution spatiale. De plus, une centaine d'images par an est au minimum exploitable à moyenne résolution spatiale. Ces données sont inversées en MES grâce à des relations définies sur le terrain. Le protocole actuel de validation doit être révisé afin de mieux estimer la qualité des quantités des MES restituées. En revanche, les gradients et la variabilité temporelle des MES sont deux indicateurs qualitatifs de la distribution spatiale des MES dans la Gironde qui sont correctement établis par le protocole RIVERCOLOR. Enfin, la détection des nuages et de leurs ombres reste encore à affiner pour mieux garantir la qualité des pixels déduits de l'analyse.

\section{Remerciements}

Nos remerciements vont au CNES, qui finance le projet RIVERCOLOR, le consortium MAGEST et EPOC, pour nous permettre de participer aux missions SOGIR. 
Thème 3 - Instrumentation, mesures, imagerie et télédétection

\section{Références bibliographiques}

DOXARAN D. (2002). Télédétection et modélisation numérique des flux sédimentaires dans l'estuaire de la Gironde. Thèse de Doctorat, Université Bordeaux 1.

DOXARAN D., FROIDEFOND J.-M., CASTAING P. (2002). A reflectance band ratio used to estimate suspended matter concentrations in the sediment-dominated coastal waters. International Journal of Remote Sensing, Vol. 23, pp 5079-5085. http://dx.doi.org/10.1080/0143116021000009912

DOXARAN D., FROIDEFOND J.-M., CASTAING P. (2003). Remote sensing reflectance of turbid sediment- dominated waters. Reduction of sediment type variations and changing illumination conditions effects using reflectance ratios. Applied Optics, Vol. 42, pp 2623-2634. http://dx.doi.org/10.1364/AO.42.002623

DOXARAN D., FROIDEFOND J.-M., CASTAING P., BABIN M. (2009). Dynamics of the turbidity maximum zone in a macrotidal estuary (the Gironde, France): Observations from field and MODIS satellite data. Estuarine, Coastal and Shelf Science, Vol. 81, pp 321-332. http://dx.doi.org/10.1016/j.ecss.2008.11.013

ETCHEBER H., SCHMIDT S., SOTTOLICHIO A., MANEUX E., CHABAUX G., ESCALIER J.M., WENNEKES H., DERRIENNIC H., SCHMELTZ M., QUEMENER L., REPECAUD M., WOERTHER P., CASTAING P., (2010). Monitoring water quality in estuarine environments: lessons from the MAGEST monitoring programme in the Gironde fluvial-estuarine system. Hydrology and Earth Systems Science Discussions, Vol. 7, pp 9411-9436. http://dx.doi.org/10.5194/hessd-7-9411-2010

HAGOLLE O., HUC M., VILLA PASCUAL D., DEDIEU G. (2010). A multi-temporal method for cloud detection, applied to FORMOSAT-2, VEN $\mu S$, LANDSAT and SENTINEL-2 images. Remote Sensing of Environment, Vol. 114, $\mathrm{n}^{\circ}$ 8, pp 1747-1755. http://dx.doi.org/10.1016/j.rse.2010.03.002

MUELlER J.L., ZANEVELD J.R.V., PEGAU S., VALDEZ E., MASKE H., ALVARAREZ-BORREGO S., LARA-LARA R. (1997). Remote sensing reflectance: preliminary comparisons between in-water and above-water measurements, and estimates modeled from measured inherent optical properties. In: Ocean Optics XIII, S.G. Ackleson, ed., Proc. SPIE 2693, pp 502-507.

SOTTOLICHIO A., CASTAING P., ETCHEBER H., MANEUX E., SCHMELTZ M., SCHMIDT S. (2011). Observations of suspended sediment dynamics in a highly turbid macrotidal estuary, derived from continuous monitoring. Journal of Coastal Research, Vol. SI 64, pp 1579-1583.

VERMOTE E. F., KOTCHENOVA S. Y., RAY J. P. (2011). MODIS Surface Reflectance User's Guide, Version 1.3.

WOERTHER P., GROUHEL, A. (1998). MAREL : Automated measurement network for the coastal environment. IEEE, Ocean's 98, Nice, pp 1149-1154. 